\title{
AN ON-CHIP HERMETIC PACKAGING TECHNOLOGY FOR MICROMECHANICAL DEVICES
}

\author{
Steve T. Cho ${ }^{1}$ and Francis M. Erdmann ${ }^{2}$ \\ ${ }^{1}$ The Charles Stark Draper Laboratory, 555 Technology Sq, Cambridge, MA 02139 \\ currently with: Abbott Laboratories, 755 Jarvis Dr, Morgan Hill, CA 95037 \\ ${ }^{2}$ Boeing North American, Inc., 3370 Miraloma Ave, Anaheim, CA 92803
}

\begin{abstract}
A novel on-chip hermetic packaging technology utilizing electrostatic bonding and eutectic sealing is presented. Planarization of the lead transfers is not required since the leads conform to the interfacial layer by forming a eutectic seal with the bonding layer (amorphous polysilicon). The leads also have an isolation resistance of $>4 G \Omega / s q$. This approach requires only one masking step and exhibits low induced stress, high thermal shock resistance, and a leak rate of $<10^{-16}$ SCCM.
\end{abstract}

\section{INTRODUCTION}

In many applications related to biological, physical, and inertial sensors, there is a need to hermetically seal the sensor onchip. Encasing the sensor in a miniature shell has several advantages, including small size, low packaging cost, and great robustness. For manufacturing applications, an inherent problem with suspended micromechanical structures is protecting the die during assembly. By encapsulating the device in a low cost shell, standard processes such as wafer sawing can be performed without yield loss.

Some of the key performance challenges in developing this technology include: i) low leak rate, ii) transferring leads from inside the package to external connections (i.e., lead transfer), iii) low temperature sealing, iv) small package size, v) induced package stress, and vi) low cost. Current approaches include anodic bonding, glass frit seals, deposited plug seals, deposited lead transfers, and thin film seals [1-4]. Although each of these techniques is adequate for sealing transducers, each approach has a significant drawback. Vacuum frit seals require large areas $(>1 \mathrm{~mm}$ width) and the choice of metals is limited due to potential dissolution during sealing. Deposited plug seal structures have limited strength over large areas and there is the potential to deposit material inside the package during sealing. Metal seals (e.g., Au-Si, In) require dielectric isolation for the lead transfers and these isolation layers require planarization; the strength of these seals is also typically less than electrostatic or frit seals. Polysilicon-toglass anodic seals with planarized polysilicon lead transfers have demonstrated hermetic seals with great robustness. However, this process also requires planarization, and for applications which require long lead transfers (e.g., flat panel displays), the resistance

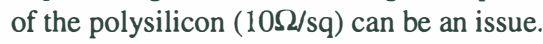

This paper presents a novel hermetic sealing technology that meets the performance goals of on-chip packaging. By integrating the techniques of anodic bonding and eutectic sealing, an on-chip seal with a leak rate of $<10^{-16}$ SCCM has been demonstrated. This seal is formed by electrostatically bonding amorphous polysilicon over gold. The anodic bond forms an atomic scale seal over the glass regions, while the gold migrates into the polysilicon to form a eutectic seal. During the sealing process, the gold flows into any microvoids that may exist; the compression due to the anodic bonding also forms a planar boundary layer. The shell material is made out of glass, which offers several advantages over silicon shells, including low stray capacitance and transparency for inspection (Fig. 1). Electrically isolating the metal leads is achieved by the high resistance of the undoped amorphous polysilicon. The process temperature can be biased far below the eutectic temperature $\left(363^{\circ} \mathrm{C}\right)$ due to localized joule heating around the metal areas. This process is simple and only requires 1 masking step. Packaged devices utilizing resonant comb drive structures have been fabricated and tested. The packages were characterized for leak rate (change in $\mathrm{Q}$ or pressure over time), lead isolation, seal strength, and thermal shock.

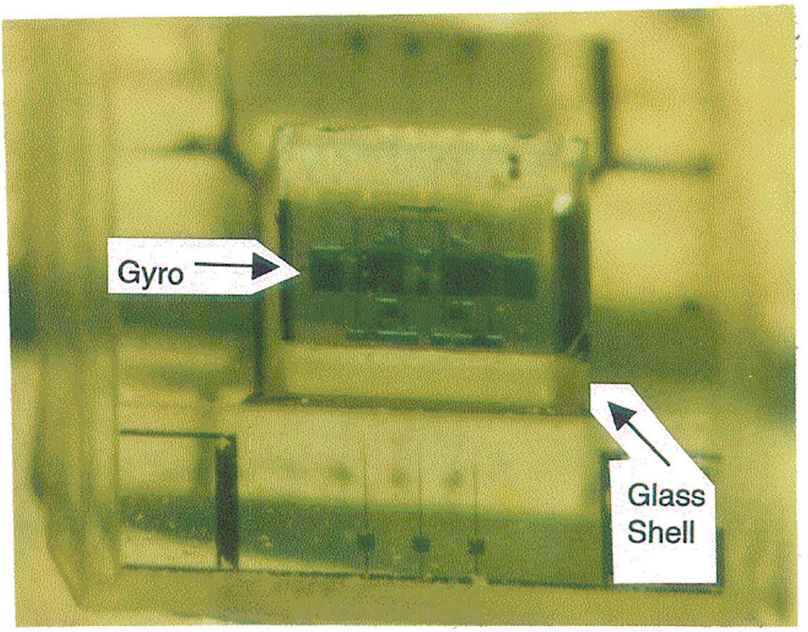

Fig. 1: Packaged Micro-Gyroscope.

In applying this approach to vacuum packaging, the key issue is outgassing. It was previously reported that the evolution of oxygen-based gases during electrostatic sealing prevented vacuum sealing and the presence of a getter was required [1]. However, by preconditioning and electrostatically outgassing the parts before bonding, vacuum seals were achieved. A proof-of-concept test was run on membrane test structures; if the membranes collapsed, then the vacuum was maintained inside the cavity. This technique can potentially reduce outgassing to low enough levels that package lifetime can be extended over 10 years. 


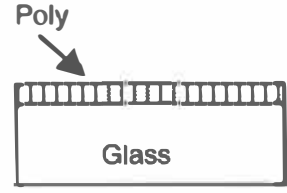

1. Deposit Polysilicon (<580 deg C).

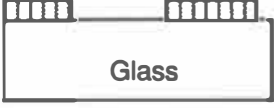

2. Pattern Recess

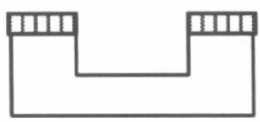

3. Etch Recess

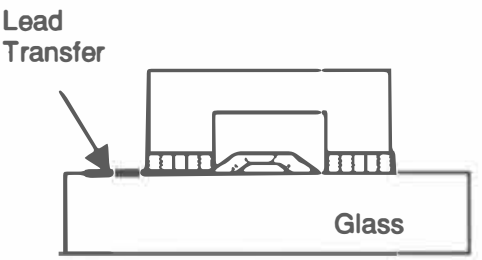

4. Bond Cap
Fig. 2: Process Flow for an On-Chip Glass Shell.

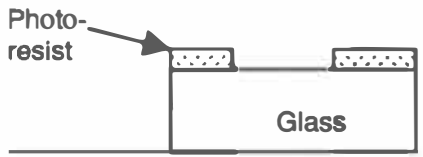

1. Pattem for Deposition

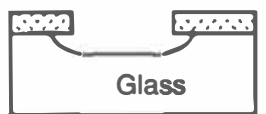

2a. Wet Etch Recess

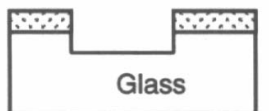

2b. Dry Etch Recess
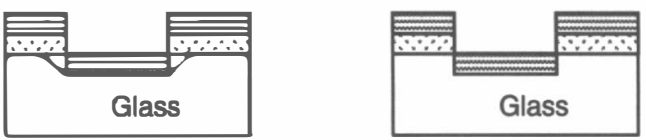

3. Deposit Metal

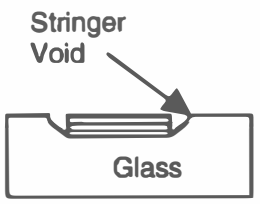

4. Lift-Off

Fig. 3: Self-Aligned Metallization Process.

\section{PROCESS DEVELOPMENT}

The ideal on-chip package should have the following characteristics: low cost, simple batch processing, high seal strength, low induced stress, low temperature sealing, and isolated lead transfers. Figure 2 shows the process flow for the shell. An amorphous structured polysilicon film is deposited on a pyrex 7740 glass wafer (far below the softening point of $821^{\circ} \mathrm{C}$ ). The amorphous structure is important for two reasons: high electrical resistance and low surface roughness. The poly is then patterned and etched in a plasma reactor. Using the poly as a mask, a deep recess is etched into the glass using an HF-based solution.

The lead transfers are run on the transducer substrate. A selfaligned lift-off process is used (Fig. 3) to define the metal. A photoresist pattern is defined on the glass and shallow recess is etched into the glass by RIE [5]. A Ti-Pt-Au layer is then deposited and lifted off. The cap is anodically bonded to the surface of the transducer. During bonding, the gold migrates into the silicon and also flows into any microvoids that exist at the Si-glass-Au interface. The RIE step is important for the lead transfer structure; wet etched recesses generated "stringer" voids at the gold-glass interface (Fig. 2a, 4). The gold migration did not consistently fill these voids. The bias temperature of the process is below the $\mathrm{Si}-\mathrm{Au}$ eutectic $\left(363^{\circ} \mathrm{C}\right)$; localized joule heating raises the bond temperature above the diffusion point.

Figure 5 shows a cross-section of the bond. During glass breaking, the interface most likely to shear was the glass-Ti area. Smooth and planar bond interfaces are evident, and the gold migration extends through the polysilicon film. The gold also fills in any potential interfacial voids that may exist.

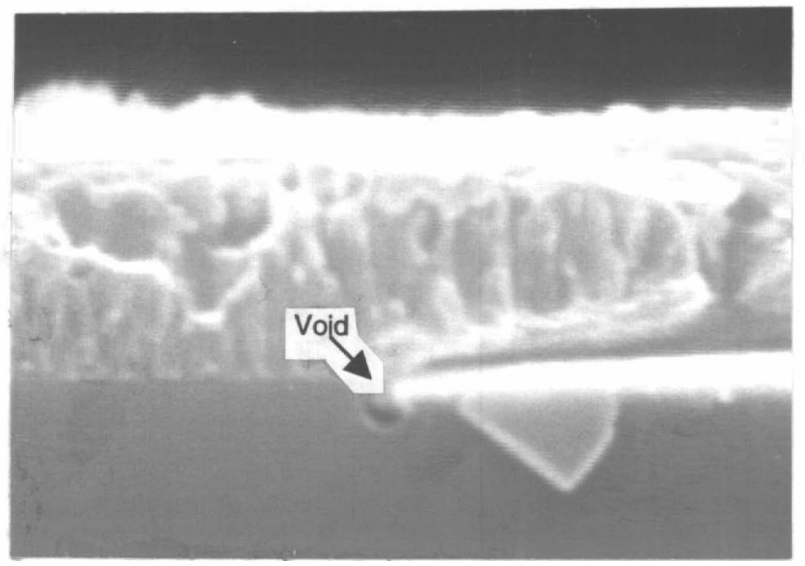

Fig. 4: Stringer Void Generated by Wet Etching.

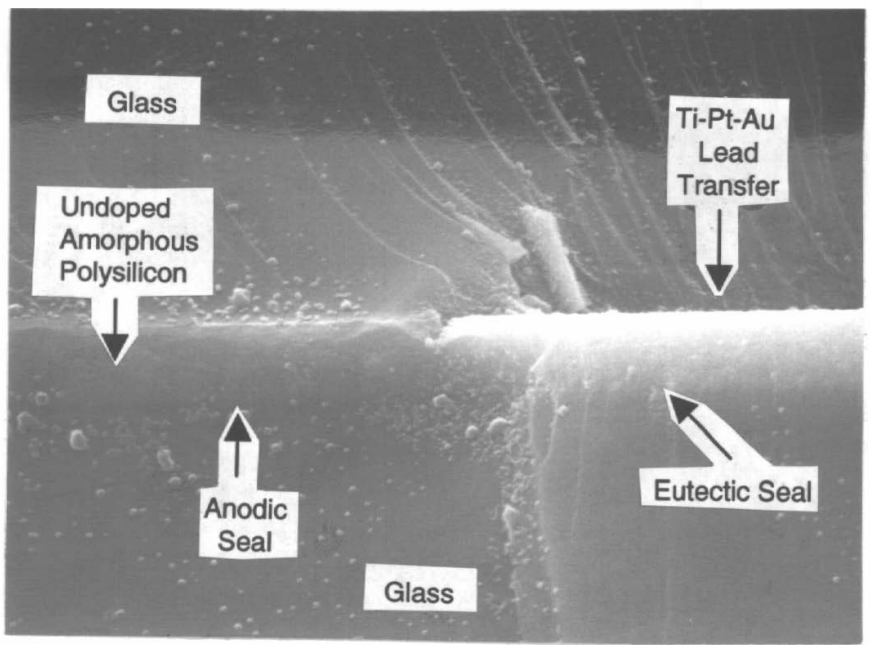

Fig. 5: Cross-Section of Bond Region. 


\section{CHARACTERIZATION}

\section{Induced Stress}

In order to measure any induced stress by the package, micromechanical resonant gyroscopes were sealed in on-chip shells. The resonant frequency before sealing was $>25,000 \mathrm{~Hz}$; the change in frequency after sealing was $<200 \mathrm{~Hz}$. Since all the materials (poly, silicon, 7740 glass) have close thermal expansion coefficient matches, the small shift was expected. For this application, the shift does not affect performance.

\section{Hermeticity}

Leak rates are defined by the pressure loss multiplied by the package volume per unit time. Atmospherically sealed shells were placed in a vacuum vessel (luTorr) and monitored for 2 weeks for changes in quality factor $(Q)$. Since $Q$ is a function of pressure, the change in pressure can be measured (Fig. 6). The measured $Q$ was 210 which corresponds to pressure of about 370 Torr (the part was sealed under atmosphere; however, subsequent cooling dropped the pressure to about half the bond ambient pressure).

After two weeks, the change in $\mathrm{Q}$ was 1 (this is within the noise of the measurement); a $\Delta \mathrm{Q}$ of 1 corresponds to a pressure change of about luTorr. Therefore, for a package volume of $2.4 \times 10^{-4} \mathrm{~cm}^{3}$, the total leak rate is $1.6 \times 10^{-17} \mathrm{SCCM}$. However, at this low level, helium permeation, ambient outgassing, and other factors will dominate the vacuum level.

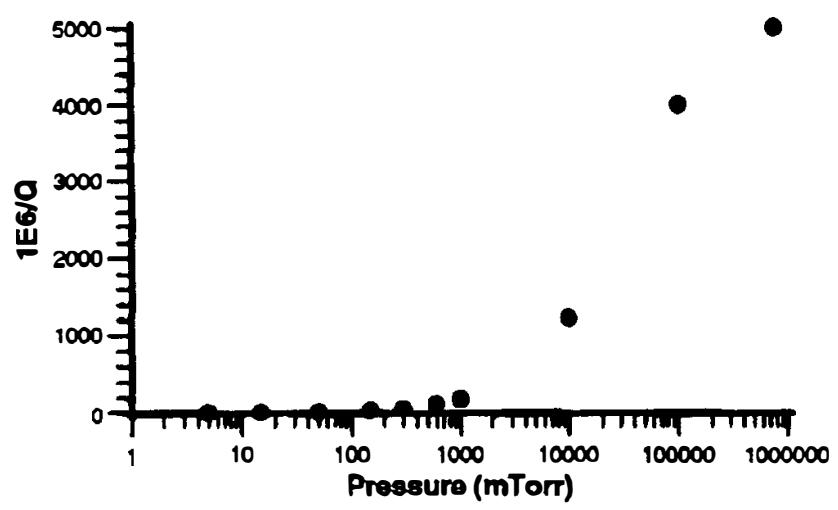

Fig. 6: Quality Factor $(Q)$ versus Pressure.

\section{Lead Transfer}

The isolation between the lead transfers was characterized. Using resistance structures of different length, the resistance of the amorphous poly was measured at $4.5 \pm 0.5 \mathrm{G} \Omega / \mathrm{sq}$. Higher temperature polysilicon films $\left(625^{\circ} \mathrm{C}\right)$ that are undoped typically have 1-2 G $\Omega /$ sq of resistance. Figure 7 shows an I-V plot of a resistance measurement; the response is linear, indicating that the gold has made good ohmic contact to the poly.

This resistance can be increased by more than an order of magnitude. By reducing the thickness of the poly and narrowing the width of the seal, the isolation resistance can be raised to $>50 \mathrm{G} \Omega / \mathrm{sq}$. Whether this is adequate depends on the noise level that can be tolerated by the application and the density of the lead transfers. However, it should be noted that typical printed circuit board resistance is $100 \mathrm{M} \Omega-1 \mathrm{G} \Omega / \mathrm{sq}$.

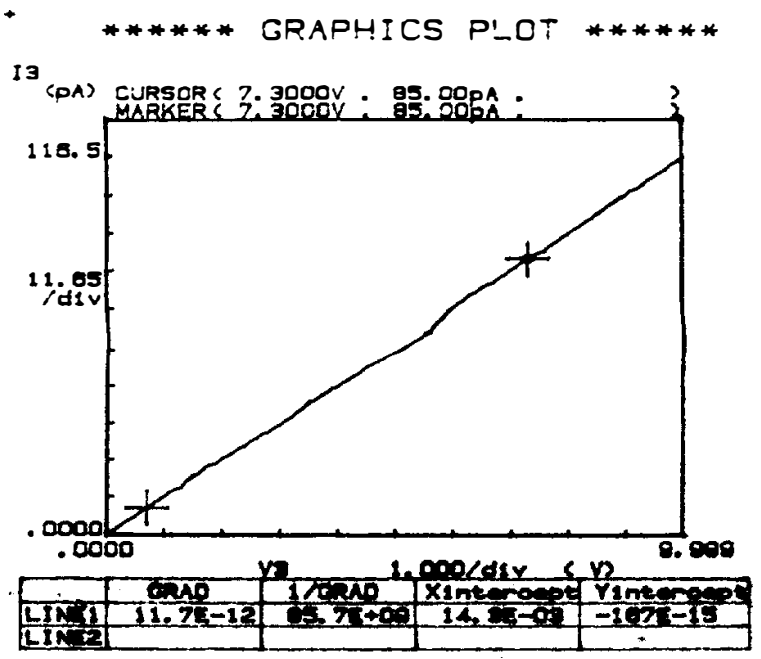

Fig. 7: I-V Plot Measuring Lead Isolation. Resistance is $4.3 \mathrm{G} \Omega / \mathrm{sq}(85.7 \mathrm{G} \Omega / 20 \mathrm{sq})$.

\section{Mechanical Robustness and Area}

The mechanical and thermal stability of the package was tested. In pull tests of the glass, the fractured or the Ti layer pulled off, but the interfacial bonds remained intact. This is consistent with traditional Si-glass anodic bonds; the bond interface is stronger than the bulk materials. The shell adhesion was also sufficiently strong enough to withstand wafer sawing. Due to the high seal strength, the bond width can be made much less than most technologies, such a frit seals.

A thermal shock test was also performed. The samples were first cooled in liquid nitrogen, then immediately placed in a convection oven operating at $150^{\circ} \mathrm{C}$. The samples maintained their mechanical integrity and hermeticity. The equivalent thermal shock is $4152^{\circ} \mathrm{K} / \mathrm{min}$.

\section{Vacuum Hermetic Sealing}

When vacuum sealing a vessel, there are several factors that determine the vacuum level, including virtual leaks, gettering, outgassing during sealing, and outgassing over the lifetime of the package. Virtual leaks are normally not a critical issue with micromechanical devices since non-porous, high vacuum materials are being used. Although lithographic getters have been developed for micro-applications [7], getter process compatability, size, and activation are primary concerns. Furthermore, the small volumes inside micromechanical packages limit the getter volume and the getter capacity. Non-getter vacuum sealing has been achieved by low temperature sealing (e.g., indium at $<150^{\circ} \mathrm{C}$ ); this low temperature is critical since outgassing rates are logarithmic.

The key issue in on-chip non-getter vacuum sealing is outgassing. Outgassing during sealing determines the initial pressure of the package and outgassing within the package determines the package lifetime. Given a package with a surface area of $1.2 \mathrm{~mm} \times 2.0 \mathrm{~mm}$, a $100 \mathrm{um}$ high cap, a bond time of 20 minutes, an outgas rate of $10^{-7}$ liter-Torr $/ \mathrm{cm}^{2}-\mathrm{sec}$, and a sealing pressure ambient of <luTorr, this would give an initial pressure which is marginally acceptable for most resonant applications. The outgas rate is dependent on how the parts are preconditioned before sealing; the rate of $10^{-7}$ liter- Torr $/ \mathrm{cm}^{2}-\mathrm{sec}$ is considered to be nominal. Glasses typically have a room temperature outgas rate of $10^{-10}$ liter-Torr $/ \mathrm{cm}^{2}$-sec; pyrex has a peak outgas rate at about $315^{\circ} \mathrm{C}$ 
[8]. If the lifetime of the package is considered to be double the initial pressure and assuming that the outgas rate does not decrease over time, then the room temperature lifetime of this package is 15 days. However, if the outgas rate could be decreased by 3 orders of magnitude, then lifetimes of up to 40 years can be achieved.

Outgassing of parts is typically performed under high vacuum (uTorr-nTorr) conditions at elevated temperatures for several hours. For high volume, high throughput applications, these practices may not be acceptable. Electrostatics offer the potential of accelerating the outgas process by liberating surface species under the influence of high electric fields. During electrostatic bonding, the depletion of oxygen-based species extends far beyond the surface of the glass.

A proof of concept test was run using membrane structures (Fig. 8) with small volumes ( $2 \mathrm{~mm} \times 2 \mathrm{~mm}, 1$.0um gap) which would be sensitive to outgassing. The first set of parts was not outgassed and bonded at $315^{\circ} \mathrm{C}$ for $\mathrm{lh}$ at luTorr. The membranes buckled slightly away from glass substrate. The second set of parts were electrostatically outgassed. A spacer separated the silicon and glass to prevent bonding. The parts had several kV's applied for one hour, then bonded at the same $315^{\circ} \mathrm{C}$ in a luTorr ambient. The membrane pressed against the bottom substrate, indicating vacuum. More research is being performed to understand and quantify the electrostatic outgassing behavior. The performance of this technology is summarized in Table 1 .

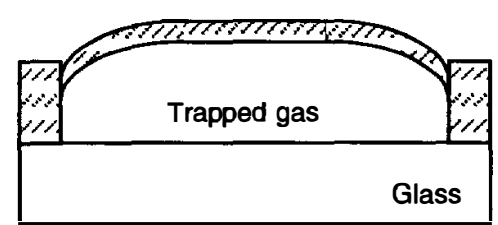

1. Membrane deflection bows out no vacuum seal.

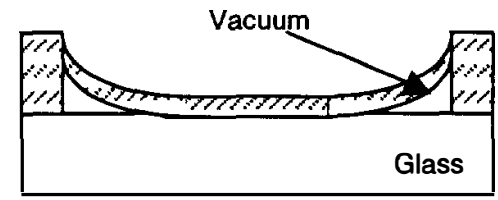

2. Membrane buckles down vacuum seal.

Fig. 8: Vacuum Sealing Test Structures.

Table 1: Hermetic Sealing Performance Summary

\begin{tabular}{|l|c|}
\hline \multicolumn{1}{|c|}{ Parameter } & Performance \\
\hline $\begin{array}{l}\text { Pressure Change (2 weeks) } \\
\text { - Leak Rate }\end{array}$ & $\begin{array}{c}<1 \mathrm{uT} \\
<10^{-16} \mathrm{SCCM}\end{array}$ \\
\hline Seal Strength & $>20 \mathrm{MPa}$ \\
\hline Lead Isolation Resistance & $4-5 \mathrm{G} \Omega / \mathrm{sq}$ \\
\hline Thermal Shock & $4152^{\circ} \mathrm{K} / \mathrm{min}$. \\
\hline
\end{tabular}

\section{CONCLUSIONS}

In many micromechanical applications related to transducers and manufacturing, there is a need to hermetically package devices on-chip. Although several approaches have been proposed, there are significant trade-offs with regards to size, cost, process complexity, and robustness. A novel sealing technology based on anodic and eutectic bonding has been presented which offers a simple, low cost approach to batch hermetic packaging. The packages are robust (mechanical strength, thermal shock) and can support vacuum sealing. Lead transfers are isolated by an amorphous polysilicon thin film with a resistance of $4.5 \mathrm{G} \Omega / \mathrm{sq}$. An electrostatic outgas approach has been presented which can potentially eliminate the use of getters in a 10 year lifetime package.

\section{ACKNOWLEDGEMENTS}

The authors would like to thank Dave Warren of Boeing, Connie Cardoso and James Cousens of Draper Labs; Paul Maciel of US Incorp., George Hopple of Candescent Technologies, and Dr. Khalil Najafi of the University of Michigan.

\section{REFERENCES}

[1] K. Minami, T. Moriuchi, and M. Esashi, "Cavity Pressure Control for Critical Damping or Packaged Micromechanical Devices", Transducers '95, pp. 240-243.

[2] S.A. Audet and K.M. Endfeld, "Integrated Sensor Level Packaging", Transducers '97, pp. 287-289.

[3] B. Ziaie, J. Von Arx, M. Dokmeci, and K. Najafi, "A Hermetic Glass-Silicon Micropackage with High Density On-Chip Feedthroughs for Sensors and Actuators", JMEMS, pp. 166-179, Vol. 5, No. 3, Sept 1996.

[4] L. Lin et al, "Vacuum Encapsulated Micro-resonators" Transducers '93, p. 270, 1993.

[5] S.T. Cho, "A Batch Dissolved Wafer Process for Low Cost Sensor Applications”, Meeting of the SPIE, Vol. 2639, pp. 10-15, October 1995.

[6] S.T. Cho, K. Najafi, and K.D. Wise, "Internal Stress Compensation and Scaling of Ultrasensitive Boron-Doped Silicon Membranes", IEEE Trans Electron Devices, Vol. 39, No. 4, pp. 836-842, April 1992.

[7] Private Communication, SAES Getters, Milan, Italy.

[8] A. Roth, Vacuum Sealing Techniques, Pergamon Press, 1968. 